\title{
Construction and analysis of a cDNA library from yellow-fruit ginseng (Panax ginseng C.A.Meyer.) leaf tissue
}

\author{
Chengjun Yang ${ }^{1,2}$, Jun Wang ${ }^{2,3 *}$, Chuanping Yang $^{2}$ and Guifeng Liu ${ }^{2}$ \\ ${ }^{1}$ Key Laboratory of Forest Tree Genetic Improvement and Biotechnology of Ministry of Education, Northeast Forestry \\ University, Harbin 150040, P. R. China. \\ ${ }^{2}$ College of Food Science and Nutritional Engineering, China Agricultural University, Beijing 100083, P. R. China.
}

Accepted 9 July, 2009

\begin{abstract}
The total RNA was isolated from yellow-fruit ginseng (Panax ginseng C.A. Meyer) leaf tissue. A cDNA library of panax ginseng leaves was constructed by using pDNR-LIB vector according to the SMART cDNA library construction kit protocol. We obtained 378 high quality sequences (GenBank accession number: ES672876-ES673253). ESTs were annotated, analyzed by BlastX and functional classified based on gene ontology, the results showed that 221 ESTs showed significant similarities to gene sequences in $\mathrm{Nr}$ database and were known genes, 21 ESTs were non-significance and unknown function genes, and 136 ESTs were considered novel genes. Most of the ESTs appeared to be related to physiological and cellular processes.
\end{abstract}

Key words: cDNA library, expressed sequence tags (EST), Panax ginseng.

\section{INTRODUCTION}

Ginseng (Panax ginseng C. A. Meyer), a perennial herb from the Araliaceae family, is one of the most commonly utilized medicinal plants. Ginseng is considered to be one of the most potent medicinal plants that have been used to bolster immunity, provide nutrition, ameliorate fatigue and enhance resistance to stress, disease and exhausttion. Ginsenosides, which are triterpene glycosides (saponins), are believed to be the main active compounds in ginseng tissues (Choi et al., 2005; Kim et al., 2006). More than 30 different ginsenosides have been isolated from ginseng plants (Sun et al., 2001; Zheng et al., 2001). Despite the considerable commercial interest in ginsenosides, little is known about the genes and biochemical pathways of ginsenoside biosynthesis. 'Jilin yellow-fruit ginseng' is identified as a homozygous recessive mutant from ordinary panax ginseng and contains high levels of ginsenosides, rich proteins, amino acids

*Corresponding author. E-mail: junwang1966@yahoo.com.cn. Tel: 86-045-82191829(O). Fax: 86-451-82190607-11. and other nutrients (Zhao et al., 1998).

cDNA library is one of the basic means to study functional genomics. Expressed sequence tags (ESTs) are partial sequences of randomly selected complementary DNA (cDNA) clones; automated sequencing techniques make it possible to generate large numbers of EST at one time. Expressed sequence tags (EST) analysis is an effective method to discover novel genes and investigate gene expression in different organs and tissues (Wang et al., 2006). The generation and analysis of expressed sequence tags provides useful information on development, metabolism and signaling in various organisms. Expressed sequence tags have applications in the discovery of new genes, mapping of the genome and identification of coding regions in genomic sequences (Miyahara et al., 2000).

In the study, we constructed a cDNA library of yellowfruit ginseng leaf tissue and obtained 378 high quality EST sequences. Through EST analysis and gene ontology, this will help us to understand the gene expression pattern, discover novel genes and study the biochemical pathways of ginsenoside biosynthesis. 


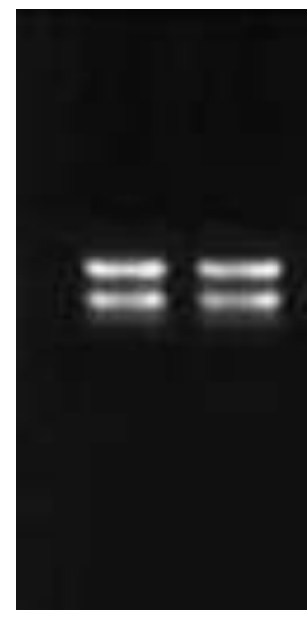

$28 \mathrm{~S}$

$18 \mathrm{~S}$

Figure 1. Agarose gel electrophoresis of total RNA from yellow-fruit ginseng leaf.

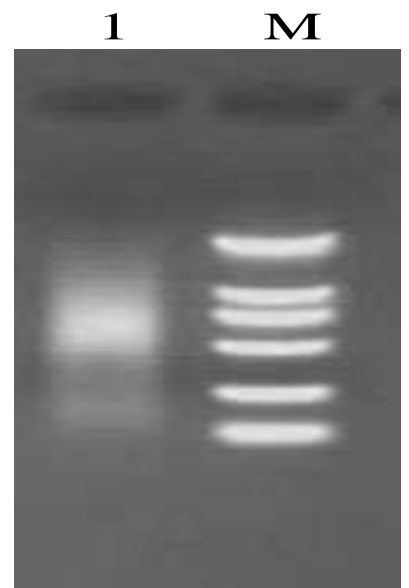

Figure 2. Agarose gel electrophoresis of doublestranded cDNA. Lane 1: double-strand cDNA; lane M: DL2000 marker.

\section{MATERIALS AND METHODS}

\section{Plant materials}

Actively growing 2 year old yellow-fruit ginseng (Panax ginseng $\mathrm{C}$. A. Meyer) leaves were obtained from ZuoJia, Jilin province, China, on June 20,2006 . The harvested leaves were immediately frozen in liquid nitrogen and then stored at $-70^{\circ} \mathrm{C}$ until RNA isolation.

\section{Isolation and quantification of RNA}

The total RNA was isolated from ginseng leaf tissue using SDS methods, as described by Yang et al. (2008). Total RNA was quantified by measuring the optical density of a dilute RNA solution. The integrity of the RNA was analyzed using $1.1 \%$ agarose/EtBr gel electrophoresis. The purity of the RNA was checked by the ratio of $\mathrm{OD}_{260} / \mathrm{OD}_{280}$.

\section{cDNA synthesis and library construction}

In accordance with the creator ${ }^{\mathrm{tm}}$ SMART ${ }^{\mathrm{TM}}$ CDNA library construction kit user manual provided by the manufacturers (Clontech), total RNA $(1.0 \mu \mathrm{g})$ as starting material was reverse transcribed to synthesize first-strand cDNA, and double-strand cDNA was synthesis by LD-PCR. $5 \mu \mathrm{g}$ of the double-stranded cDNA were taken for analysis by electrophoresis on a 1.1\% agarose/EtBr gel. Then the amplified double-strand cDNA was digested with proteinase $\mathrm{K}$ and Sfi I. After digestion, cDNA size fractionation was performed using chroma spin-400 columns to collect cDNA large than $400 \mathrm{bp}$ and checked the profile of fractions on a $1.1 \%$ agarose/EtBr gel. The cDNA was ligated to the Sfi I-digested dephosphorylated pDNR-LIB vector provided with the kit and electroporated into DH5a Escherichia coli bacteria to develop the cDNA library of ginseng leaf tissue. To make a large, stable quantity of a high-titer stock of the library, we amplified the primary cDNA library.

\section{Identification of the cDNA library}

According to the library tittering protocol, the unamplified and amplified cDNA library were tittered. To identify the cDNA inserts of the recombinants and determined the percentage of recombinant clones, 16 plaques were randomly picked from plate. Then PCR was performed with $\mathrm{M} 13$ primers provided by the advantage 2 PCR kit. The PCR products checked on 1.2\% TAE/agarose gel with DNA size markers.

\section{Library sequencing and analysis}

The cDNA library clones were plated into LB agar plate containing $30 \mu \mathrm{g} / \mathrm{ml}$ of chloramphenicol, white clones were picked in to 96 well plates randomly, plasmids DNA of each clone were prepared by standard alkaline lysis preparation protocol and then sequenced by Beijing genomics institute. Sequencing was performed in a Mega BACE1000 DNA capillary sequence machine. The raw expressed sequence tags (EST) were edited to remove vector and poor quality sequences. The remaining sequences were subjected to blast analysis against the non-redundant database on the GeneBank (http://www.ncbi.nlm.nih.gov/blast) for similarity. The confirmed sequences were submitted to the dbEST database of GeneBank. Identified genes were classified according to gene ontology. The network information of the gene ontology database is categorized into 3 groups: cellular component, molecular function and biological process.

\section{RESULTS AND DISCUSSION}

High-quality total RNA was isolated from the leaf tissue of ginseng. Electrophoresis of the total RNA on $1.1 \%$ agarose/EtBr showed distinct 28S and 18S rRNA bands and the ratio of intensities of $28 \mathrm{~S}$ and $18 \mathrm{~S}$ was about $2: 1$ (Figure 1), the ratio of $O D_{260} / O_{280}$ to the total RNA was 1.90. The total RNA isolated was integrated and suitable for constructing the cDNA library. The double-strand cDNA synthesized using LD-PCR was analyzed on a $1.1 \%$ agarose $/ \mathrm{EtBr}$ gel and the product showed a smear from 0.2 to $2.5 \mathrm{~kb}$ (Figure 2). The double-strand cDNA fractionated using CHROMA SPIN-400 columns was 


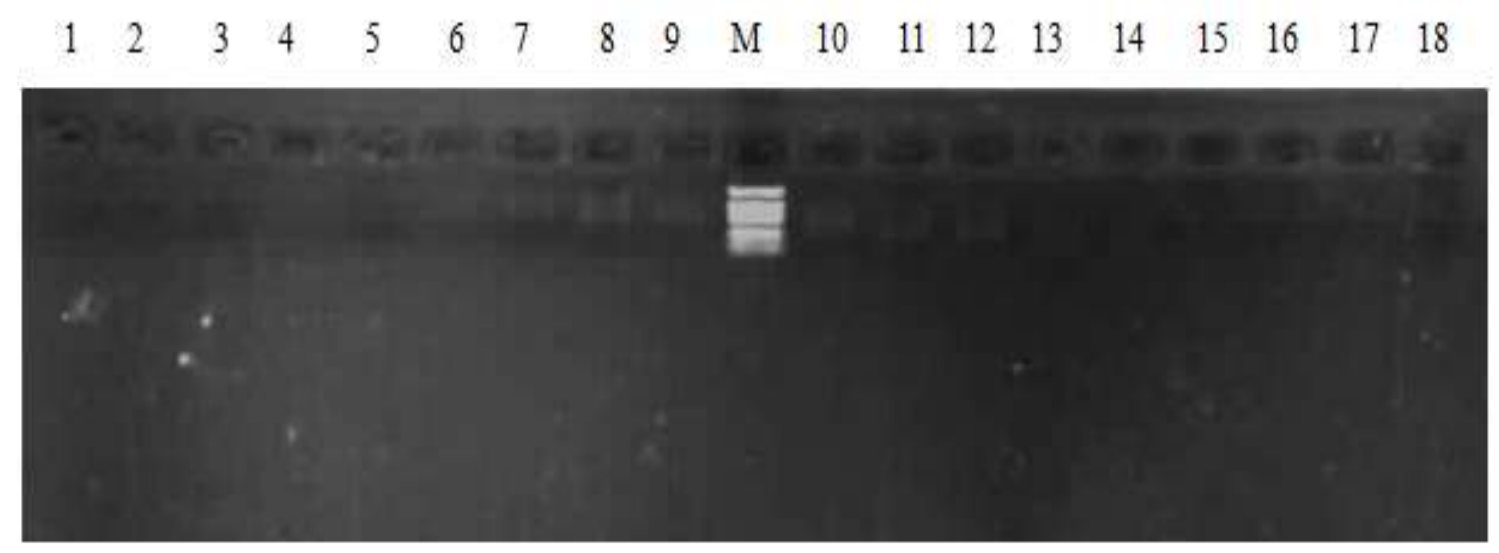

Figure 3. Double-stranded cDNA fractiionated on a 1.1\% agarose/EtBr gel 1-18: double-stranded cDNA; M: DL2000 marker.

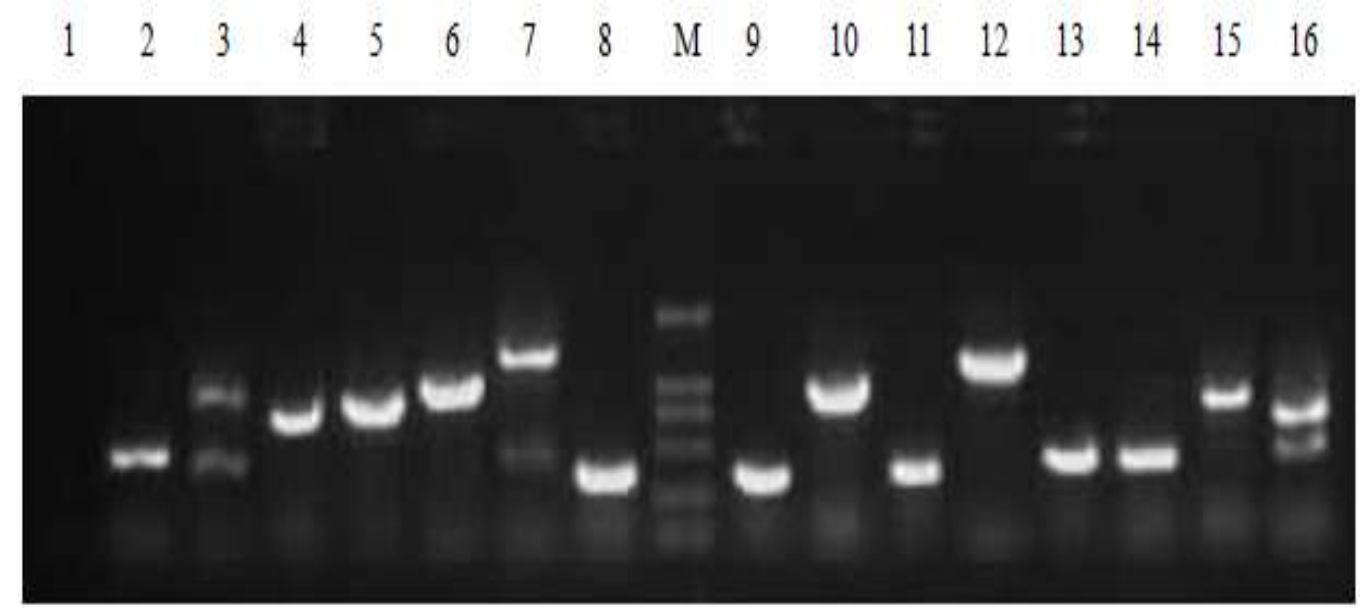

Figure 4. 16 clones in the cDNA library were selected randomly to evaluate their insert sizes. Lanes 116: Products of inserts; lane M: DL2000 marker.

collected and pipette $3 \mu \mathrm{l}$ of cDNA to run on a $1.1 \%$ agarose/EtBr gel at $150 \mathrm{~V}$ for $10 \mathrm{~min}$. Electrophoresis results showed the cDNA in lanes 7 - 11 was larger than $400 \mathrm{bp}$ (Figure 3) and was ready to be ligated to the Sfi Idigested, dephosphorylated pDNR-LIB vector.

The titer of the un-amplified constructed cDNA libray was approximately $1.01 \times 10^{6} \mathrm{pfu} / \mathrm{ml}$, the titer of the amplified cDNA library was $2.16 \times 10^{9} \mathrm{pfu} / \mathrm{ml}$. 16 independent clones were selected randomly from unamplified cDNA library and were amplified by PCR to check the percentage of recombinants and the insert size of the recombinants. The results showed the percentage of recom-binants was $93.75 \%$, the cDNA inserts ranged from 0.3 to $2 \mathrm{~kb}$ (Figure 4), with an average size of 700 bp. The results indicated that the quality of cDNA library should be sufficient to identify the expressed genes in yellow-fruit ginseng.

A total of 400 randomly selected clones were sequenced from the library. Of these, 378 high quality sequences were obtained after deletion of the vector sequences and sequences with short base pairs. The obtained 378 EST sequences were submitted to GeneBank (accession No: ES672876-ES673253) and dbEST (ID: 46881386 46881763). Through BlastX analysis, 221ESTs showed significant similarities to gene sequences in $\mathrm{Nr}$ database and were known genes, 21 ESTs were non-significance and unknown function genes, and 136 ESTs, which have no matched were considered novel genes in $P$. giseng. The results of BLASTX search and annotation are shown in Table 1. The generated EST were categorized using gene ontology terms as shown in Table 2, which provide a structured vocabulary to describe a sequence according to its cellular component status, molecular function and biological process. Most EST appeared to be related to physiological and cellular processes.

In conclusion, we described the construction of a cDNA library from yellow-fruit ginseng leaf tissue, EST analysis and gene ontology. The results of analysis of $378 \mathrm{cDNA}$ 
Table 1. List of BlastX searched and annotated expressed sequence tags from yellow-fruit ginseng leaf cDNA library.

\begin{tabular}{|c|c|c|c|c|c|}
\hline $\begin{array}{c}\text { Accession } \\
\text { oo. }\end{array}$ & $\begin{array}{c}\text { Identity } \\
(\%)\end{array}$ & $\begin{array}{c}\text { Score } \\
\text { (bit) }\end{array}$ & E-value & Annotation & $\begin{array}{l}\text { No of } \\
\text { EST }\end{array}$ \\
\hline ES672914 & 92 & 310 & $1.00 \mathrm{E}-83$ & (S)-2-hydroxy-acid oxidase (EC 1.1.3.15) - cucurbit & 1 \\
\hline ES673235 & 87 & 61.2 & $2.00 \mathrm{E}-10$ & $19 S$ proteasome regulatory complex subunit S6A & 1 \\
\hline ES673125 & 81 & 66.6 & $3.00 \mathrm{E}-10$ & 1-aminocyclopropane-1-carboxylate oxidase & 1 \\
\hline ES673214 & 80 & 279 & $3.00 \mathrm{E}-74$ & 1-deoxyxylulose 5-phosphate synthase & 1 \\
\hline ES672956 & 86 & 303 & $4.00 \mathrm{E}-81$ & $60 S$ ribosomal protein $\mathrm{L} 13$ & 1 \\
\hline ES673025 & 97 & 186 & $3.00 \mathrm{E}-46$ & $60 S$ ribosomal protein L37a & 1 \\
\hline ES672934 & 79 & 183 & $3.00 \mathrm{E}-45$ & GTP binding & 1 \\
\hline ES673044 & 84 & 183 & $2.00 \mathrm{E}-45$ & AT-HF & 1 \\
\hline ES672970 & 100 & 253 & $1.00 \mathrm{E}-66$ & ATP synthase beta subunit & 1 \\
\hline ES673152 & 93 & 63.2 & $8.00 \mathrm{E}-12$ & ATP synthase CF-0 subunit I & 1 \\
\hline ES672877 & 100 & 174 & $6.00 \mathrm{E}-43$ & ATPase epsilon subunit & 3 \\
\hline ES672903 & 73 & 227 & 7.00E-59 & putative AtRer1A protein & 1 \\
\hline ES672922 & 83 & 150 & $1.00 \mathrm{E}-35$ & auxin response factor 3 & 1 \\
\hline ES672960 & 68 & 184 & $1.00 \mathrm{E}-45$ & blight-associated protein $\mathrm{p} 12$ precursor & 2 \\
\hline ES673234 & 78 & 241 & 5.00E-63 & carbonate dehydratase/ zinc ion binding & 1 \\
\hline ES673212 & 94 & 133 & $1.00 \mathrm{E}-30$ & CAB-like protein [Ipomoea nil] & 1 \\
\hline ES673022 & 71 & 163 & 1.00E-39 & calcium ion binding & 2 \\
\hline ES673029 & 60 & 156 & 4.00E-37 & calcium-binding protein & 1 \\
\hline ES672945 & 48 & 98.2 & 7.00E-20 & Ribonuclease Mc1 & 1 \\
\hline ES672901 & 67 & 151 & $1.00 \mathrm{E}-35$ & chitinase-like protein & 1 \\
\hline ES672917 & 99 & 275 & 8.00E-73 & chlorophyll a/b binding protein & 3 \\
\hline ES673014 & 99 & 295 & 4.00E-79 & chlorophyll a/b binding protein of LHCIl type I precursor & 5 \\
\hline ES673007 & 94 & 193 & $2.00 \mathrm{E}-48$ & Chlorophyll a-b binding protein 13 , chloroplast precursor & 1 \\
\hline ES673114 & 92 & 112 & $9.00 \mathrm{E}-49$ & Chlorophyll a-b binding protein, chloroplast precursor & 1 \\
\hline ES672969 & 90 & 134 & $9.00 \mathrm{E}-31$ & chloroplast hypothetical protein & 2 \\
\hline ES672953 & 64 & 217 & $2.00 \mathrm{E}-55$ & chloroplast oxygen-evolving enhancer protein & 2 \\
\hline ES673194 & 86 & 304 & $1.00 \mathrm{E}-81$ & chloroplast pigment-binding protein CP24 & 1 \\
\hline ES673148 & 56 & 75.1 & $7.00 \mathrm{E}-13$ & conserved hypothetical protein & 1 \\
\hline ES672983 & 70 & 213 & $2.00 \mathrm{E}-54$ & CONSTANS-like protein & 1 \\
\hline ES673055 & 78 & 132 & $5.00 \mathrm{E}-30$ & copper chaperone & 1 \\
\hline ES672886 & 70 & 270 & 7.00E-72 & CPD photolyase & 1 \\
\hline ES673171 & 89 & 296 & 3.00E-79 & cyclophilin & 1 \\
\hline ES673041 & 100 & 303 & $1.00 \mathrm{E}-81$ & cytoplasmic ribosomal protein $\mathrm{S} 13$ & 1 \\
\hline ES672961 & 64 & 132 & $5.00 \mathrm{E}-30$ & dehydrin 4 & 1 \\
\hline ES673219 & 76 & 85.1 & $7.00 \mathrm{E}-16$ & delta 12 oleic acid desaturase FAD2 & 1 \\
\hline ES673113 & 67 & 199 & $3.00 \mathrm{E}-50$ & Desiccation protectant protein Lea14 homolog & 2 \\
\hline ES673180 & 95 & 316 & $3.00 \mathrm{E}-85$ & DSK2 & 1 \\
\hline ES673046 & 77 & 195 & $7.00 \mathrm{E}-49$ & electron transporter/ thiol-disulfide exchange intermediate & 1 \\
\hline ES672999 & 77 & 164 & $1.00 \mathrm{E}-39$ & elongation factor 1 -beta & 1 \\
\hline ES672981 & 71 & 115 & $5.00 \mathrm{E}-25$ & enoyl-CoA-hydratase & 1 \\
\hline ES673040 & 71 & 54.3 & $1.00 \mathrm{E}-06$ & expressed protein & 1 \\
\hline ES672915 & 82 & 253 & $9.00 \mathrm{E}-67$ & ferritin & 1 \\
\hline ES673057 & 75 & 253 & $2.00 \mathrm{E}-66$ & galactinol synthase, isoform GolS-1 & 1 \\
\hline ES673002 & 100 & 95.5 & $5.00 \mathrm{E}-19$ & GBR3 & 3 \\
\hline ES673076 & 39 & 84.7 & $1.00 \mathrm{E}-15$ & GBR5 & 5 \\
\hline ES673205 & 73 & 192 & $3.00 \mathrm{E}-48$ & GDSL-lipase protein & 1 \\
\hline ES673079 & 94 & 105 & $3.00 \mathrm{E}-22$ & geranylgeranyl reductase & 1 \\
\hline ES673091 & 83 & 60.1 & 3.00E-08 & glossy1 homolog & 1 \\
\hline
\end{tabular}


Table 1. contd.

\begin{tabular}{|c|c|c|c|c|c|}
\hline ES672967 & 88 & 215 & $7.00 \mathrm{E}-55$ & glutathione peroxidase & 1 \\
\hline ES673220 & 84 & 66.6 & $2.00 \mathrm{E}-10$ & glyceraldehyde 3-phosphate dehydrogenase & 1 \\
\hline ES673215 & 78 & 220 & 2.00E-56 & glyceraldehyde 3-phosphate dehydrogenase B subunit & 1 \\
\hline ES673075 & 84 & 278 & 5.00E-74 & glyceraldehyde-3-phosphate dehydrogenase & 1 \\
\hline ES672997 & 52 & 109 & $5.00 \mathrm{E}-23$ & Harpin-induced 1 & 1 \\
\hline ES673176 & 81 & 171 & $1.00 \mathrm{E}-41$ & heat-shock protein 80 & 1 \\
\hline ES673042 & 46 & 145 & $9.00 \mathrm{E}-34$ & histone acetyltransferase complex component & 1 \\
\hline ES673020 & 75 & 124 & $1.00 \mathrm{E}-27$ & hypothetical protein & 1 \\
\hline ES672963 & 67 & 164 & 2.00E-39 & hypothetical protein & 1 \\
\hline ES673016 & 47 & 65.9 & 4.00E-10 & hypothetical protein Afu4g09870 & 1 \\
\hline ES673132 & 85 & 52 & 1.00E-07 & hypothetical protein CIMG_06034 & 1 \\
\hline ES673048 & 92 & 123 & 4.00E-27 & hypothetical protein MtrDRAFT_AC151668g11v1 & 17 \\
\hline ES673230 & 92 & 106 & 2.00E-37 & hypothetical protein MtrDRAFT_AC151668g27v1 & 1 \\
\hline ES673043 & 88 & 131 & 8.00E-30 & hypothetical protein OeelhCp020 & 2 \\
\hline ES673035 & 81 & 91.3 & $1.00 \mathrm{E}-17$ & hypothetical protein OeelhCp021 & 1 \\
\hline ES673010 & 97 & 169 & $4.00 \mathrm{E}-41$ & hypothetical protein PhapfoPp090 & 2 \\
\hline ES673039 & 63 & 121 & $1.00 \mathrm{E}-26$ & hypothetical protein SNOG_04123 & 1 \\
\hline ES673162 & 73 & 77 & $2.00 \mathrm{E}-13$ & late embryogenis abundant protein 5 & 1 \\
\hline ES673151 & 99 & 340 & 3.00E-92 & light harvesting chlorophyll a /b binding protein & 7 \\
\hline ES673197 & 88 & 167 & $2.00 \mathrm{E}-40$ & Single-stranded DNA-binding protein & 1 \\
\hline ES672906 & 58 & 57.4 & $1.00 \mathrm{E}-07$ & low temperature and salt responsive protein & 1 \\
\hline ES673232 & 78 & 206 & 1.00E-52 & ly200 protein & 1 \\
\hline ES673061 & 56 & 151 & $9.00 \mathrm{E}-36$ & Major sperm protein & 1 \\
\hline ES673185 & 74 & 243 & $2.00 \mathrm{E}-63$ & malate dehydrogenase & 1 \\
\hline ES673017 & 43 & 62 & $1.00 \mathrm{E}-08$ & metal ion binding & 1 \\
\hline ES672955 & 75 & 86.7 & $4.00 \mathrm{E}-16$ & metallothionein- 1 like protein & 1 \\
\hline ES672883 & 82 & 82.4 & $6.00 \mathrm{E}-15$ & mRNA-binding protein precursor & 1 \\
\hline ES673068 & 61 & 77.4 & $1.00 \mathrm{E}-13$ & NAK-type protein kinase & 1 \\
\hline ES673078 & 64 & 136 & 7.00E-31 & nonspecific lipid transfer protein 1 & 2 \\
\hline ES672938 & 74 & 143 & 2.00E-33 & O-GlcNAc-transferase-like protein & 1 \\
\hline ES673139 & 93 & 61.2 & $1.00 \mathrm{E}-08$ & putative GMPase & 1 \\
\hline ES673119 & 86 & 193 & $2.00 \mathrm{E}-54$ & ALM beta-like & 1 \\
\hline ES673000 & 50 & 158 & $8.00 \mathrm{E}-38$ & putative adapter-related protein complex 4 epsilon 1 subunit & 1 \\
\hline ES673030 & 78 & 53.1 & 3.00E-06 & putative lipase & 1 \\
\hline ES673155 & 61 & 147 & 2.00E-34 & early flowering 4 & 1 \\
\hline ES673242 & 50 & 62.4 & $6.00 \mathrm{E}-16$ & hypothetical protein & 1 \\
\hline ES673141 & 76 & 75.1 & $9.00 \mathrm{E}-13$ & oxidoreductase & 1 \\
\hline ES673071 & 94 & 296 & $1.00 \mathrm{E}-79$ & oxygen evolving complex $33 \mathrm{kDa}$ photosystem II protein & 1 \\
\hline ES673240 & 39 & 59.7 & 3.00E-08 & $16 \mathrm{kDa}$ protein of the photosynthetic oxygen- evolving protein & 1 \\
\hline ES673161 & 89 & 54.7 & $1.00 \mathrm{E}-06$ & PAP fibrillin & 1 \\
\hline ES673051 & 62 & 75.5 & $6.00 \mathrm{E}-13$ & peptidase/ threonine endopeptidase & 1 \\
\hline ES673237 & 88 & 270 & $1.00 \mathrm{E}-71$ & permease & 1 \\
\hline ES673033 & 90 & 117 & $1.00 \mathrm{E}-25$ & peroxiredoxin $Q$ & 1 \\
\hline ES673054 & 35 & 58.9 & 7.00E-08 & phloem protein 2-1 & 4 \\
\hline ES672921 & 39 & 66.2 & $5.00 \mathrm{E}-10$ & phloem protein 2-2 & 9 \\
\hline ES673208 & 89 & 238 & 7.00E-62 & Phosphoribulokinase & 1 \\
\hline ES673053 & 100 & 183 & $1.00 \mathrm{E}-45$ & photosynthetic electron transfer-like protein & 1 \\
\hline ES673207 & 80 & 196 & $2.00 E-49$ & photosystem I subunit XI & 1 \\
\hline ES673104 & 45 & 75.1 & $1.00 \mathrm{E}-12$ & photosystem II & 1 \\
\hline ES672994 & 71 & 168 & $2.00 \mathrm{E}-41$ & photosystem II $10 \mathrm{kDa}$ protein & 3 \\
\hline
\end{tabular}


Table 1. contd.

\begin{tabular}{|c|c|c|c|c|c|}
\hline ES673116 & 41 & 72.8 & $5.00 \mathrm{E}-12$ & Photosystem II 5 kDa protein, chloroplast precursor (PSII-T) & 1 \\
\hline ES673227 & 100 & 56.6 & 3.00E-07 & photosystem II CP47 protein & 1 \\
\hline ES673177 & 100 & 39.7 & $3.00 \mathrm{E}-06$ & photosystem II M protein & 2 \\
\hline ES673239 & 87 & 200 & $1.00 \mathrm{E}-50$ & photosystem II protein D1 & 2 \\
\hline ES673252 & 94 & 105 & 7.00E-22 & photosystem II protein $\mathrm{K}$ & 1 \\
\hline ES673146 & 63 & 125 & 4.00E-28 & plastidic aldolase NPALDP1 & 1 \\
\hline ES672927 & 52 & 95.1 & $1.00 \mathrm{E}-18$ & plastoquinol-plastocyanin reductase & 2 \\
\hline ES673221 & 92 & 140 & $2.00 \mathrm{E}-32$ & poly(A)-binding protein & 2 \\
\hline ES672984 & 84 & 79 & $5.00 \mathrm{E}-14$ & Polygalacturonase-1 non-catalytic subunit beta precursor (AroGP1) & 1 \\
\hline ES673015 & 81 & 184 & $2.00 \mathrm{E}-45$ & protein binding / ubiquitin-protein ligase/ zinc ion binding & 1 \\
\hline ES672980 & 84 & 144 & $2.00 \mathrm{E}-33$ & protein disulfide isomerase & 1 \\
\hline ES673203 & 86 & 59.7 & 4.00E-08 & PsbC & 1 \\
\hline ES673005 & 98 & 178 & $4.00 \mathrm{E}-44$ & PSI 9 kDa protein & 1 \\
\hline ES673027 & 68 & 53.5 & 2.00E-06 & PSI-H precursor & 1 \\
\hline ES672992 & 100 & 75.5 & $7.00 \mathrm{E}-13$ & PSII 44 kDa protein & 1 \\
\hline ES672925 & 100 & 96.3 & $3.00 \mathrm{E}-19$ & PSII K protein & 1 \\
\hline ES672896 & 52 & 112 & 8.00E-24 & structural constituent of ribosome & 1 \\
\hline ES672978 & 72 & 221 & $2.00 \mathrm{E}-56$ & putative $60 \mathrm{~S}$ ribosomal protein L7-like protein & 1 \\
\hline ES673059 & 82 & 232 & $6.00 \mathrm{E}-60$ & Putative auxin efflux carrier component 8 (AtPIN8) & 1 \\
\hline ES673135 & 91 & 161 & $9.00 \mathrm{E}-39$ & putative chlorophyll A-B binding protein of LHCl type II precursor & 1 \\
\hline ES673200 & 98 & 115 & $4.00 \mathrm{E}-25$ & putative chloroplast chlorophyll A-B binding protein type I & 1 \\
\hline ES672923 & 94 & 77.4 & $1.00 \mathrm{E}-13$ & putative chloroplast thiazole biosynthetic protein & 1 \\
\hline ES672946 & 76 & 157 & $3.00 \mathrm{E}-37$ & putative $\mathrm{E} 2$, ubiquitin-conjugating enzyme UBC7 & 1 \\
\hline ES672943 & 41 & 93.6 & $2.00 \mathrm{E}-18$ & putative F-box and leucine-rich repeat protein & 1 \\
\hline ES673154 & 76 & 213 & $2.00 \mathrm{E}-54$ & putative L24 ribosomal protein & 1 \\
\hline ES673236 & 61 & 140 & $2.00 \mathrm{E}-32$ & putative phosphatidylcholine-sterol acyltransferase & 1 \\
\hline ES672880 & 80 & 169 & $4.00 \mathrm{E}-41$ & $\begin{array}{l}\text { putative phosphatidylinositol- phosphatidylcholine transfer protein } \\
\text { SEC14 }\end{array}$ & 1 \\
\hline ES673217 & 74 & 187 & $7.00 \mathrm{E}-47$ & putative photosystem I reaction centre PSI-D subunit precursor & 2 \\
\hline ES673011 & 76 & 172 & $5.00 \mathrm{E}-42$ & putative rubisco subunit binding-protein alpha subunit & 2 \\
\hline ES673112 & 54 & 164 & $1.00 \mathrm{E}-39$ & receptor-like protein kinase homolog RK20-1 & 1 \\
\hline ES673244 & 81 & 202 & $5.00 \mathrm{E}-51$ & Ribonuclease 1 & 3 \\
\hline ES672907 & 100 & 181 & $7.00 \mathrm{E}-45$ & ribosomal protein L16 & 1 \\
\hline ES672876 & 83 & 106 & $3.00 \mathrm{E}-22$ & Ribosomal protein S30 & 1 \\
\hline ES673003 & 78 & 114 & $1.00 \mathrm{E}-24$ & Ribosomal protein S5, bacterial and organelle form & 1 \\
\hline ES672940 & 94 & 99.8 & 4.00E-20 & ribosomal protein small subunit 28 & 2 \\
\hline ES673123 & 94 & 298 & $5.00 \mathrm{E}-83$ & ribulose-1,5-bisphosphate carboxylase/oxygenase activase & 2 \\
\hline ES673006 & 98 & 342 & $3.00 \mathrm{E}-93$ & ribulose-1,5-bisphosphate carboxylase/oxygenase small subunit & 3 \\
\hline ES673175 & 45 & 135 & $5.00 \mathrm{E}-31$ & RNA-binding protein-like & 1 \\
\hline ES673201 & 90 & 80.1 & $3.00 \mathrm{E}-14$ & rRNA intron-encoded homing endonuclease & 1 \\
\hline ES673065 & 93 & 67.8 & $9.00 \mathrm{E}-16$ & S-adenosylmethionine synthetase & 1 \\
\hline ES673173 & 93 & 69.3 & $1.00 \mathrm{E}-15$ & selenium binding & 1 \\
\hline ES672919 & 88 & 209 & $3.00 \mathrm{E}-53$ & small GTP-binding protein & 1 \\
\hline ES672924 & 75 & 88.6 & $8.00 \mathrm{E}-17$ & sorbitol related enzyme & 1 \\
\hline ES673074 & 72 & 102 & $5.00 \mathrm{E}-21$ & structural constituent of ribosome & 2 \\
\hline ES673143 & 60 & 94.7 & $1.00 \mathrm{E}-18$ & T-complex protein 1 epsilon subunit & 1 \\
\hline ES673064 & 46 & 103 & $3.00 E-25$ & TGF-beta receptor, type I/II extracellular region & 1 \\
\hline ES672993 & 49 & 118 & $1.00 \mathrm{E}-25$ & thioredoxin $\mathrm{H}$ & 2 \\
\hline ES673225 & 74 & 150 & $1.00 \mathrm{E}-35$ & Thioredoxin H-type (TRX-H) emb|CAA94534.1| thioredoxin & 1 \\
\hline ES672979 & 60 & 128 & $6.00 \mathrm{E}-29$ & TIR-NBS disease resistance-like protein [Populus trichocarpa] & 1 \\
\hline
\end{tabular}


Table 1. contd.

\begin{tabular}{|l|l|l|l|l|c|}
\hline ES672944 & 80 & 224 & $1.00 \mathrm{E}-57$ & tonoplast intrinsic protein & 1 \\
ES673110 & 63 & 156 & $4.00 \mathrm{E}-37$ & vacuolar ATPase subunit E-like protein & 1 \\
ES673164 & 76 & 80.9 & $2.00 \mathrm{E}-14$ & type 2 metallothionein & 1 \\
unknown & & & & & 21 \\
no & & & & & 136 \\
\hline matched & & & & & 378 \\
\hline \multicolumn{1}{|c|}{ Total } & & & & & \\
\hline
\end{tabular}

Table 2. Gene ontology of expressed sequence tags from yellow-fruit ginseng leaf cDNA library.

\begin{tabular}{|l|c|}
\hline \multicolumn{1}{|c|}{ Gene ontology term } & No. of genes \\
\hline Cell component & 46 \\
\hline Cell & 46 \\
Cell part & 20 \\
Organelle & 8 \\
Organelle part & 26 \\
Protein complex & \\
\hline Molecular function & 1 \\
\hline Antioxidant activity & 30 \\
Binding & 25 \\
Catalytic activity & 2 \\
Enzyme regulator activity & 2 \\
Molecular function unknown & 5 \\
Obsolete molecular function & 1 \\
Signal transducer activity & 13 \\
Structural molecule activity & 1 \\
Translation regulator activity & 8 \\
Transporter activity & \\
\hline Biological process & 65 \\
\hline Cellular process & 75 \\
Physiological process & 2 \\
Regulation of biological process & 4 \\
Response to stimulus & \\
\hline
\end{tabular}

demonstrated EST sequencing and data analysis as a useful and efficient approach to identifying novel genes and for functional genes expression, which would help us understand the mechanisms of ginseng plants during development stages and enrich our knowledge of functional genomic research in ginseng.

\section{ACKNOWLEDGEMENT}

This work was supported by a grant from State Forestry Administration, P.R. China (010-413255).

\section{REFERENCES}

Choi DW, Jung JD, Ha YI, Park HW, In DS, Chung HJ, Liu JR (2005). Analysis of transcripts in methyl jasmonate-treated ginseng hairy roots to identify genes involved in the biosynthesis of ginsenosides and other secondary metabolites Plant Cell Rep. 23: 557-566

Kim MK, Lee BS, In JG, Sun H, Yoon JH, Yang DK (2006). Comparative analysis of expressed sequence tags (ESTs) of ginseng leaf. Plant Cell Rep. 25: 599-606

Miyahara T, Hirono I, Aoki T (2000). Analysis of expressed sequence tags from a Japanese eel Anguilla japonica spleen cDNA library. Fish. Sci. 66: 257-260

Sun YL, Xue WZ (2001). Study on the main chemical constituents in ginseng. Chin. J. Health Lab. Technol. 5: 555-556. (in chinese.)

Wang YC, Yang CP, Liu GF, Jiang J, Wu JH (2006). Generation and analysis of expressed sequence tags from a cDNA library of Tamarix androssowii. Plant Sci. 170: 28-36

Yang CJ, Wang J, Zhou L, Liu GJ (2008). Extraction of total RNA from Panax ginseng C.A. Meryer. cv. Hongguo leaves. Biotechnol. Bull. 1: 136-139. (in chinese.)

Zhao SJ, Liu YZ, Zhao YH, Li FY, Huang ZH, Wu LJ, Guo J, Liu JY (1998). Comprehensive evaluation on characters of Jilin yellow-fruit ginseng. Special wild economic animal plant res. 4: 1-6. (in chinese.)

Zheng YL, Zhang CX, Li XG (2001). Indicators and methods of quality evaluation to Jilin ginseng. Ginseng res. 2: 12-14. (in chinese.) 